\title{
EDITORIAL
}

\section{Angiogenesis and apatinib: a new hope for patients with advanced gastric cancer?}
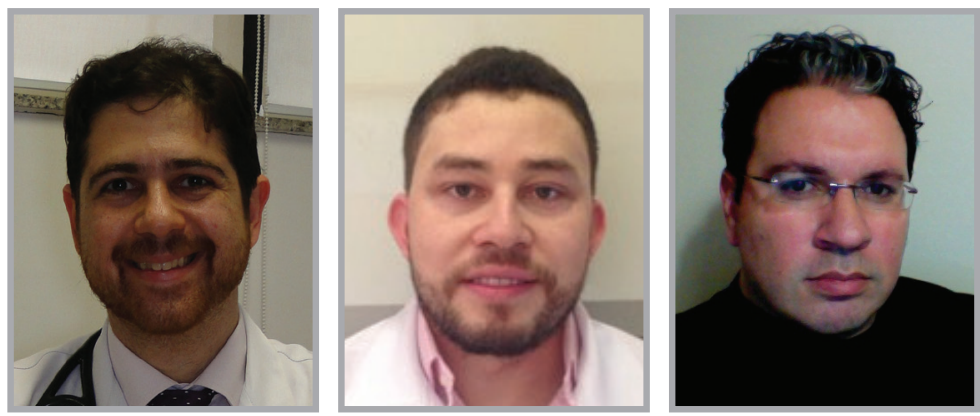

Ramon Andrade de Mello*,2,2,3 Jailson de Oliveira ${ }^{3} \&$ Georgios Antoniou ${ }^{4}$

First draft submitted: 6 July 2016; Accepted for publication: 7 September 2016; Published online: 22 September 2016

Gastric cancer (GC) is a highly aggressive disease with a high incidence and an ominous prognosis, particularly in China, Brazil and some Mediterranean European countries [1]. Several environmental factors are involved in GC pathogenesis, in addition to genetic predisposition [1-3]. It is well known that lifestyle attitudes, such as high alcohol intake, tobacco consumption and spicy food, result in an increased susceptibility for GC [2]. Screening program strategies are not easy to establish in occidental countries, which has resulted in a late diagnosis at the advanced stage of the disease for the majority of GC cases. Over the past decade, approaches toward GC have been changing, particularly in the metastatic setting. Platinum-fluoropyrimidine chemotherapy, however, remains the backbone for systemic treatment $[1,4]$. Furthermore, a set of patients with a good performance status may benefit from triplet regimens that include taxane [1,5]. More recently, molecular therapies have emerged with an additive effect, similar to that used with other tumor types (e.g., lung, melanoma) [6-9]. Trastuzumab (a monoclonal antibody against HER2), when added to standard platinum-fluoropyrimidine chemotherapy, showed an improved overall survival (OS) benefit in HER2-expressing (as identified by immunohistochemistry) GC patients $[1,10]$. Angiogenesis has been shown to play an active role in GC pathogenesis; hence, antiangiogenics have demonstrated benefits in the advanced setting $[3,6,11-12]$, with the urokinase plasminogen activating system and VEGF recently being suggested to contribute synergistically to both tumor progression and aggressiveness $[9,13]$. In addition, HIF-1, a heterodimer comprising the oxygen-regulated subunit HIF- $1 \alpha$ and HIF-1 $\beta$, mediates the transcription of the gene for VEGF [13-15]. The overexpression of HIF- $1 \alpha$ is associated with tumor neo-angiogenesis and

\section{KEYWORDS}

- angiogenesis • apatinib • gastric cancer

“...in the perioperative setting, a combination of apatinib with standard platinum-fluoropyrimidine chemotherapy may result in a clinical gain in terms of response." 
“...trials in different disease settings and with a variety of these drug combinations are warranted to explore the optimal method and timing and to develop the best possible approach to provide better care for our patients." sprouting and with tumor cell proliferation and invasion [14]. The role of VEGFA, B, C, D, E and its receptors (VEGFR1, VEGFR2 and VEGFR3) in several steps of tumor angiogenesis, lymphagiogenesis, cell proliferation and metastasis are well established in the literature [3]. Interestingly, the interaction between VEGFA and VEGFR2 effectively promoted the creation of novel tumor vessels via a stronger ligand-receptor binding, which resulted in cell downregulation that favored more rapid tumorigenesis steps [2-3,9,12]. Although the interaction between VEGFA and VEGFR1 is effective, it is weaker in terms of ligand-receptor binding [8]. VEGFR3, in contrast, is more likely to be associated with lymphangiogesis $[3,8]$. Thus, several angiogenesis-targeting drugs are being evaluated in the framework of systemic treatments [11,16-21].

In May 2016, an original article by Jin $\mathrm{Li}$ and coworkers [11] reported the results of a Phase III study using apatinib (an oral tyrosine kinase inhibitor of VEGFR2) in patients with refractory gastric or gastroesophageal junction (GEJ) cancer with two or more previous lines of treatment. The authors emphasized that although GC is currently the third cause of death from cancer worldwide, there is still no standard treatment that has shown any satisfactory response or improved survival rates for those patients with refractory to chemotherapy gastric or GEJ cancer [11] .

The study was supported by previous results from the promising use of antiangiogenic drugs in the treatment of GC [21]. In one study, bevacizumab (anti-VEGF), when used as a first-line therapy in combination with chemotherapy, was associated with increased response rates but not with OS $[9,16,19]$. Another study used ramucirumab (anti-VEGFR2) as a second-line therapy and showed increased OS, especially when combined with a taxane $[19,21]$. Finally, a Phase II randomized study from 2013 found increased OS rates for GC patients who were treated with apatinib after failure of chemotherapy, thus providing adequate support for a Phase III study [21].

Despite mentioning relevant studies, the authors failed to make any reference to the seminal studies that pioneered targeted therapy for advanced GC, such as a study on anti-HER2 therapy, in which trastuzumab combined with chemotherapy was associated with increased survival in advanced GC patients who expressed HER2 $[1,10]$ or a study that discussed the major aspects of targeted therapy for advanced GC (especially anti-HER2 therapy) $[1,10,20]$.
One study [11] was designed as a multicenter, placebo-controlled, Phase III trial with 273 patients from 23 Chinese centers who were randomized at 2:1 (176 receiving apatinib $850 \mathrm{mg}$ orally, once a day and 71 receiving placebo). Randomization was used to increase the statistical power of the study because of the small sample size. The primary end points were OS and progression-free survival. The secondary outcome parameters were response rate, quality of life and safety.

The patients in the two groups were matched, which facilitated comparisons. OS was significantly longer $(+1.8$ months $)$ in the treatment group. Progression-free survival was also significantly longer with apatinib treatment (2.6 months) than with placebo treatment (1.8 months). The gain was slightly smaller than the gain in OS observed for the apatinib group in the Phase II study (2.3 months), despite the smaller sample size $(n=143)$. Thus, studies based on larger samples should be conducted to understand these differences. In the overall analysis of safety, grade 3 and 4 toxicity, hand-foot syndrome, hypertension and hematological toxicity were rarely observed.

The groups did not differ significantly in their quality of life scores at any time during the study period, which suggests that apatinib is a well-tolerated option.

The study of apatinib is relevant because no standard treatment has been established for patients with GC chemotherapy refractory disease. This is the first Phase III study [11] showing the efficacy of a well-tolerated oral antiangiogenic drug in monotherapy for GC refractory to chemotherapy.

The authors [11] concluded that in addition to being less toxic than bevacizumab, apatinib is an attractive therapeutic option for patients with GC/GEJ cancer refractory to two lines of treatment and beyond. However, the lack of any effective options in patients with advanced GC refractory to more than two lines of chemotherapy makes apatinib an interesting alternative. Its effectiveness as a first-line therapy in the metastatic setting has yet to be proven, especially because trastuzumab with 5-fluorouracil and platinum-containing chemotherapy in HER2positive patients is considered successful. The median OS could even reach 17 months, according to the ToGA trial [10]. Considering the lack of targeted drug options for HER2-negative GC patients in first-line therapy, apatinib combined 
with chemotherapy should be assessed both in this setting and as a maintenance therapy after satisfactory response to first-line chemotherapy. A well-designed clinical trial involving both patients with HER2-positive and HER2-negative disease is important to answer this question and clarify the remaining issues. Finally, the association of apatinib and standard chemotherapy should be evaluated in an adjuvant or peri-operative framework to explore its benefits in terms of being a potential curative intended therapy. We also hypothesize that in the perioperative setting, a combination of apatinib with standard platinum-fluoropyrimidine chemotherapy may result in a clinical gain in terms of response. This notion is based on the fact that a bulky, potentially resectable disease carries a high tumoral burden, which could be more exposed and thus presents a better response to targeted therapy. Nevertheless, we should also be vigilant about the possible toxicity profile of this combination, predominantly the risks of bleeding and hypertension. In conclusion, trials in different disease settings and with a variety of these drug combinations are warranted to explore the optimal method and timing and to develop the best possible approach to provide better care for our patients.

\section{Financial \& competing interests disclosure}

$R A$ de Mello is a consultantlon the advisory board for Pfizer, Zodiac; honoraria from AstraZeneca. He has received educational grants from Amgen and Pierre Fabre. The authors have no other relevant affiliations or financial involvement with any organization or entity with a financial interest in or financial conflict with the subject matter or materials discussed in the manuscript. This includes employment, consultancies, honoraria, stock ownership or options, expert testimony, grants or patents received or pending, or royalties.

No writing assistance was utilized in the production of this manuscript.

\section{References}

Papers of special note have been highlighted as: - of interest; $\bullet$ of considerable interest

1 De Mello RA, Marques AM, Araújo A. HER2 therapies and gastric cancer: a step forward. World J. Gastroenterol. 19(37), 6165-6169 (2013).

2 Luis M, Tavares A, Carvalho LS, Lara-Santos L, Araújo A, De Mello RA. Personalizing therapies for gastric cancer: molecular mechanisms and novel targeted therapies. World J. Gastroenterol. 19(38), 6383-6397 (2013).

3 Marques I, Araújo A, De Mello RA. Anti-angiogenic therapies for metastatic colorectal cancer: current and future perspectives. World J. Gastroenterol. 19(44), 7955-7971 (2013).

4 Okines A, Norman A, Mccloud P, Kang Y-K, Cunningham D. Meta-analysis of the REAL-2 and ML17032 trials: evaluating capecitabinebased combination chemotherapy and infused 5-fluorouracil-based combination chemotherapy for the treatment of advanced oesophago-gastric cancer. Ann. Oncol. 20(9), 1529-1534 (2009).

5 Van Cutsem E, Moiseyenko VM, Tjulandin S et al. Phase III study of docetaxel and cisplatin plus fluorouracil compared with cisplatin and fluorouracil as first-line therapy for advanced gastric cancer: a report of the V325 Study Group. J. Clin. Oncol. 24(31), 4991-4997 (2006).
- Reports the results of docetaxel and cisplatin plus fluorouracil in first line for advanced gastric cancer (gastric cancer [GC]; an important therapeutic option for those with good performance status).

6 Abdel-Rahman O. Targeting vascular endothelial growth factor (VEGF) pathway in gastric cancer: preclinical and clinical aspects. Crit. Rev. Oncol. Hematol. 93(1), 18-27 (2015).

7 De Mello RA, Pousa I, Pereira D. Nivolumab for advanced squamous cell lung cancer: what are the next steps? Lancet Oncol. 16(3), 234-235 (2015).

8 De Mello RA, Costa BM, Reis RM, Hespanhol V. Insights into angiogenesis in non-small cell lung cancer: molecular mechanisms, polymorphic genes, and targeted therapies. Recent Pat. Anticancer Drug Discov. 7(1), 118-131 (2012).

9 Ortega JS, Steinberg S, Moro E et al. Comparative study of tumor angiogenesis and immunohistochemistry for $\mathrm{p} 53$, c-ErbB2, c-myc and EGFr as prognostic factors in gastric cancer. Histol. Histopathol. 15(2), 455-462 (2000).

- Describes important pathways of angiogenesis in GC.

10 Bang Y-J, Van Cutsem E, Feyereislova A et al. Trastuzumab in combination with chemotherapy versus chemotherapy alone for treatment of HER2-positive advanced gastric or gastro-oesophageal junction cancer (ToGA): a Phase 3, open-label, randomised controlled trial. Lancet 376(9742), 687-697 (2010).

-• Defines the role of trastuzumab in metastatic GC.

11 Li J, Qin S, Xu J et al. Randomized, double-blind, placebo-controlled Phase III trial of Apatinib in patients with chemotherapy-refractory advanced or metastatic adenocarcinoma of the stomach or gastroesophageal junction. J. Clin. Oncol. 34(13), 1448-1454 (2016).

12 Li Q, Zhang N, Jia Z et al. Critical role and regulation of transcription factor FoxM1 in human gastric cancer angiogenesis and progression. Cancer Res. 69 (8), 3501-3509 (2009).

13 Uefuji K, Ichikura T, Mochizuki $\mathrm{H}$. Cyclooxygenase-2 expression is related to prostaglandin biosynthesis and angiogenesis in human gastric cancer. Clin. Cancer Res. 6(1), 135-138 (2000).

14 Stoeltzing O, Mccarty MF, Wey JS et al. Role of hypoxia-inducible factor $1 \alpha$ in gastric cancer cell growth, angiogenesis, and vessel maturation. J. Natl Cancer Inst. 96(12), 946-956 (2004).

15 Tu SP, Jiang XH, Lin MC et al. Suppression of survivin expression inhibits in vivo tumorigenicity and angiogenesis in gastric cancer. Cancer Res. 63(22), 7724-7732 (2003).

16 Ohtsu A, Shah MA, Van Cutsem E et al. Bevacizumab in combination with chemotherapy as first-line therapy in advanced 
EDITORIAL de Mello, de Oliveira \& Antoniou

gastric cancer: a randomized, double-blind, placebo-controlled Phase III study. J. Clin. Oncol. 29(30), 3968-3976 (2011).

- This trial shows results of bevacizumab (an antiangiogenic drug for GC).

17 Mi Y-J, Liang Y-J, Huang H-B et al. Apatinib (YN968D1) reverses multidrug resistance by inhibiting the efflux function of multiple ATP-binding cassette transporters. Cancer Res. 70 (20), 7981-7991 (2010).

18 Van Cutsem E, De Haas S, Kang Y-K et al. Bevacizumab in combination with chemotherapy as first-line therapy in advanced gastric cancer: a biomarker evaluation from the AVAGAST randomized Phase III trial. J. Clin. Oncol. 30(17), 2119-2127.

19 Wilke H, Muro K, Van Cutsem E et al. Ramucirumab plus paclitaxel versus placebo plus paclitaxel in patients with previously treated advanced gastric or gastrooesophageal junction adenocarcinoma (RAINBOW): a double-blind, randomised Phase 3 trial. Lancet Oncol. 15(11), 1224-1235 (2014).
-. Important as it shows the importance of ramucirumab in previously treated advanced GC.

20 Shah MA, Ilson D, Kelsen DP. Thromboembolic events in gastric cancer: high incidence in patients receiving irinotecan-and bevacizumab-based therapy. J. Clin. Oncol. 23(11), 2574-2576 (2005).

21 Li J, Qin S, Xu J et al. Apatinib for chemotherapy-refractory advanced metastatic gastric cancer: results from a randomized, placebo-controlled, parallel-arm, Phase II trial. J. Clin. Oncol. 31(26), 3219-3225 (2013). 